\title{
Indirect forces between impurities in one-dimensional quantum liquids
}

\author{
P. Wächter, V. Meden, and K. Schönhammer \\ Institut für Theoretische Physik, Universität Göttingen, \\ Friedrich-Hund-Platz 1, D-37077 Göttingen, Germany
}

\begin{abstract}
We investigate the indirect interaction between two isolated impurities in a Luttinger liquid described by a microscopic lattice model. To treat the electron-electron interaction $U$ the functional renormalization group method is used. For comparison we also study the $U=0$ case. We find that for a wide range of impurity parameters the impurity interaction $V_{12}$ as a function of their separation $r$ oscillates with decaying amplitude between being attractive and repulsive. For half-filling of the band and in a crossover regime between weak and strong impurities the interaction becomes purely attractive. For $U=0$ and independent of the impurity strength the amplitude of the interaction energy falls off as $1 / r$. For $U>0$ the decay for small separations and weak to intermediate impurities is governed by a $U$ dependent exponent larger than -1 , which crosses over to -1 for large $r$. The crossover scale depends on the impurity strength and $U$. We present simple pictures which explain our results in the limits of weak and strong impurities. We finally also consider attractive interactions $U<0$.

PACS numbers: 71.10.Pm, 73.21.Hb, 03.75.Ss, 03.75.Hh
\end{abstract}

\section{INTRODUCTION}

The physical properties of interacting one-dimensional (1D) electron systems differ substantially from the generic Fermi liquid behavior of higher-dimensional systems, offering a whole variety of interesting new effects. $\frac{1}{}$ Replacing the Fermi liquid concept of quasi-particles, the Luttinger liquid (LL) phenomenology has proved to capture the low-energy physics of a large class of models. ${ }^{2}$ The low lying excitations of the system are no longer described by quasi-particles, but rather by collective density excitations. Accordingly, even the presence of a single isolated impurity can have strong effects on the properties of the system $3,4,5,6,7$

For the case of two isolated impurities placed in a LL the linear conductance has been investigated in detail $.8,9,10,11,12,13$ Other aspects have not yet been discussed to this extent. Here we study the indirect interaction between two impurities mediated by the electrons. A particular promising candidate for measuring such forces are impurities in fermionic "atomic quantum wires" realized with ultracold gases $\underline{\underline{14}}$ For a continuum model of noninteracting electrons with delta impurities it was shown that the impurity interaction $V_{12}$ as a function of the impurity separation $r$ oscillates between being attractive and repulsive. Its magnitude decays as $1 / r$. The period of the oscillation is $\pi / k_{F}$, with $k_{F}$ being the Fermi momentum $\stackrel{15}{\underline{5}}$ The effect of a repulsive interaction was then taken into account using a field theoretical effective low-energy model and considering the two limits of weak and strong bare impurities $\stackrel{15,16}{1}$ The weak impurity case was analyzed using linear response theory while for strong impurities methods developed in the context of quantum Brownian motion were applied ${ }^{17}$ In addition, for strong repulsive electron-electron interactions boundary conformal field theory was used to study the impurity interaction $\underline{\underline{18}}$

Here we supplement the earlier studies. We consider a microscopic lattice model, focus on small to intermediate electron-electron interaction and apply a method which is nonperturbative in the strength of the impurities. This allows us to study the crossover from the weak to the strong impurity behavior. For the two limits simple pictures of the observed physics are presented. Some emphasis is put on the question whether the impurity interaction as a function of $r$ continues to oscillate also in the presence of electron-electron interaction ${ }^{15,16,18} \mathrm{We}$ briefly discuss the case of attractive interactions.

The method of choice for our calculations is the recently developed functional renormalization group (fRG), which has turned out to be a powerful tool in the study of low-dimensional interacting electron systems $\stackrel{19,20}{ } \mathrm{We}$ use an approximate truncation scheme that by comparison to exact and numerical results was shown to provide a good approximation for small to intermediate electronelectron interaction. It was used for interacting systems of up to $10^{7}$ lattice sites $\stackrel{13,21}{ }$

The paper is organized as follows. In Sec. II] we introduce our model and in Sec. III give a brief outline of the fRG procedure used. In Secs. [V] and $\nabla$ we present our results for the $r$ dependence of the impurity interaction considering noninteracting and interacting electrons, respectively. We establish contact to the former calculations. Finally, in Sec. VI our findings are summarized.

\section{THE MODEL}

As our microscopic model we use the lattice model of spinless fermions with nearest-neighbor hopping and nearest-neighbor interaction on a large but finite number $N$ of lattice sites. To suppress the effect of the boundaries the interacting chain is coupled to noninteracting semi-infinite leads via adiabatic contacts. They are realized by varying the interaction smoothly across the two contacts from zero to the bulk value $U \stackrel{13}{\underline{n}}$ We are mainly 
interested in the half filled band case.

The kinetic part of the Hamiltonian reads

$$
H_{\mathrm{kin}}=-t \sum_{j=-\infty}^{\infty}\left(c_{j+1}^{\dagger} c_{j}+\text { H.c. }\right),
$$

where $c_{j}^{\dagger}$ and $c_{j}$ denote the fermionic creation and annihilation operators on lattice site $j$. We set $t=1$ and use the hopping as the unit of energy. In addition, the lattice spacing is set to one. The electrons are assumed to interact on the bonds between the sites $[1, N]$ via a spatially dependent nearest-neighbor interaction

$$
H_{\mathrm{int}}=\sum_{j=1}^{N-1} U_{j, j+1}\left(n_{j}-\frac{1}{2}\right)\left(n_{j+1}-\frac{1}{2}\right),
$$

defining the interacting wire of interest. The operators on the right-hand side of Eq. (2) are the local density operators $n_{j}=c_{j}^{\dagger} c_{j}$ shifted by $-1 / 2$ to assure that the interacting part of the system is half filled. To turn on the interaction smoothly we use the function

$$
U_{j, j+1}=U \frac{\arctan \left[s\left(j-j_{s}\right)\right]-\arctan \left[s\left(1-j_{s}\right)\right]}{\arctan \left[s\left(\frac{N}{2}-j_{s}\right)\right]-\arctan \left[s\left(1-j_{s}\right)\right]}
$$

with $s=\frac{1}{4}$ and $j_{s}=56$ for the left part $\left[1, \frac{N}{2}\right]$ of the interacting wire and a similar function for the right part. In combination with the semi-infinite leads this provides us with a wide region in the center of the interacting wire, which has a constant interaction strength $U$. Hardly any effects from the contacts can be detected 13,21

In the region of constant $U$ we place the two impurities whose interaction we want to study. We mainly consider site impurities, modeled by the Hamiltonian $\left(V_{\alpha}>0\right)$

$$
H_{\mathrm{imp}}=\sum_{\alpha=1}^{2} V_{\alpha} n_{j_{\alpha}}
$$

and sketched in Fig. 1, but also use hopping impurities described by $\left(0<t_{\alpha}<1\right)$

$$
H_{\mathrm{imp}}^{\prime}=-\sum_{\alpha=1}^{2}\left(t_{\alpha}-1\right)\left[c_{j_{\alpha}+1}^{\dagger} c_{j_{\alpha}}+\text { H.c. }\right] .
$$

For both types of impurities the impurity separation $r$ is defined as $r=j_{2}-j_{1}$.

The homogeneous model $H=H_{\text {kin }}+H_{\text {int }}$ with constant interaction between all bonds (that is also in the semi-infinite leads) can be solved exactly via a Bethe ansatz ${ }^{22}$ and exhibits LL behavior for all fillings and all $U$ except for half-filling with $|U| \geq 2 \underline{23}$ In the half filled case, the relation between the LL parameter $K$, which later on will become important, and the interaction strength $U$ can be given in a closed form 22,23

$$
K^{-1}=\frac{2}{\pi} \arccos \left(-\frac{U}{2}\right) \text {. }
$$

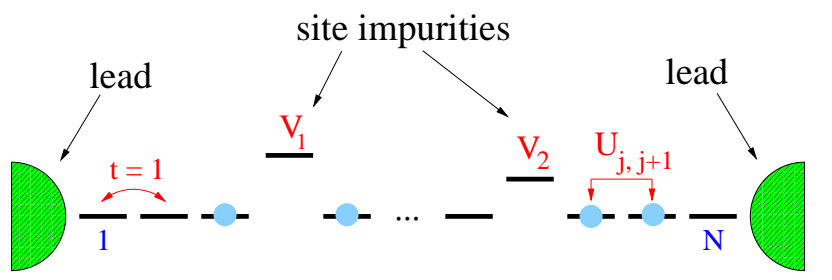

FIG. 1: (Color online) Schematic plot of the lattice model with site impurities; in the case of hopping impurities $t \neq 1$ for two bonds

\section{FUNCTIONAL RENORMALIZATION GROUP}

\section{A. General setup}

The general formulation of the fRG for the generating functional $\Gamma$ of the one-particle irreducible $n$-particle vertices starts by introducing an infrared cutoff $\Lambda$ in the free propagator leading to a $\Lambda$ dependent $\Gamma^{\Lambda}$. We here use a sharp energy cutoff $\underline{21}$ By differentiating with respect to $\Lambda$ one can derive an exact infinite hierarchy of coupled differential flow equations for the vertex functions, $\underline{19,20}$

The adaption of this general scheme to our present problem, namely to a spinless inhomogeneous LL at temperature $T=0$, and the approximations involved in deriving a closed set of equations for the self-energy are described in great detail in Ref. 21. The self-energy is approximated as frequency independent $\Sigma^{\Lambda}(i \omega) \rightarrow \Sigma^{\Lambda}$. Defining $\tilde{G}^{\Lambda}(i \omega)=\left[G_{0}(i \omega)-\Sigma^{\Lambda}\right]^{-1}$, with the Green function $G_{0}$ obtained from the kinetic part Eq. (11) of the Hamiltonian, the flow equations for the spatial (Wannier basis) matrix elements read

$$
\begin{aligned}
\partial_{\Lambda} \Sigma_{j, j}^{\Lambda} & =-\frac{1}{\pi} \sum_{r= \pm 1} U_{j, j+r}^{\Lambda} \operatorname{Re} \tilde{G}_{j, j+r}^{\Lambda}(i \Lambda), \\
\partial_{\Lambda} \Sigma_{j, j \pm 1}^{\Lambda} & =\frac{1}{\pi} U_{j, j+1}^{\Lambda} \operatorname{Re} \tilde{G}_{j, j \pm 1}^{\Lambda}(i \Lambda),
\end{aligned}
$$

with

$$
U_{j, j+1}^{\Lambda}=\frac{U_{j, j+1}}{1+\left(\Lambda-\frac{2+\Lambda^{2}}{\sqrt{4+\Lambda^{2}}}\right) \frac{U_{j, j+1}}{2 \pi}} .
$$

The 2-particle vertex was parametrized by a static nearest-neighbor interaction of strength $U_{j, j+1}^{\Lambda}$ which implies that the self-energy is a tridiagonal matrix. The flow equation (9) of the 2-particle vertex is the especially simple form for half-filling, derivable from the general formula at arbitrary filling. ${ }^{21}$ The fRG flow leads from $\Lambda=\infty$ down to $\Lambda=0$, where the original system is recovered. At the end of the flow $\Sigma_{j, j^{\prime}}^{\Lambda=0}$ present the frequency independent approximation for the self-energy. This approximation scheme to the full hierarchy of flow equations was successfully used to study various aspects of inhomogeneous LLs: $\underline{12,13,21}$

For our lattice model the matrix elements of the inverse of the full Green function at scale $\Lambda,\left[\tilde{G}^{\Lambda}\right]^{-1}(i \Lambda)$ for $j, j^{\prime} \in$ 
$[1, N]$ are given by

$$
\begin{aligned}
& {\left[\tilde{G}^{\Lambda}\right]_{j, j^{\prime}}^{-1}(i \Lambda)=i \Lambda \delta_{j, j^{\prime}}+\left(\delta_{j, j^{\prime}+1}+\delta_{j, j^{\prime}-1}\right)} \\
& -\frac{1}{2}\left(i \Lambda-\sqrt{(i \Lambda)^{2}-4}\right)\left(\delta_{j, j^{\prime}} \delta_{j, 1}+\delta_{j, j^{\prime}} \delta_{j, N}\right)-\Sigma_{j, j^{\prime}}^{\Lambda} .
\end{aligned}
$$

The second to last term effectively accounts for the semiinfinite leads as an additional contribution to the selfenergy $\stackrel{13}{=}$

The initial values at cutoff $\Lambda_{0} \gg 1$ of the fRG flow equations (77) and (8) in the case of site impurities are given by $\underline{21}$

$$
\begin{aligned}
\Sigma_{j, j}^{\Lambda_{0}} & =\sum_{\alpha=1}^{2} V_{\alpha} \delta_{j, j_{\alpha}}, \\
\Sigma_{j, j \pm 1}^{\Lambda_{0}} & =0,
\end{aligned}
$$

whereas for hopping impurities one finds

$$
\begin{aligned}
\Sigma_{j, j}^{\Lambda_{0}} & =0, \\
\Sigma_{j, j \pm 1}^{\Lambda_{0}} & =-\sum_{\alpha=1}^{2}\left(t_{\alpha}-1\right) \delta_{j, j_{\alpha}} .
\end{aligned}
$$

\section{B. 0-particle vertex and grand canonical potential}

Earlier papers using the fRG for 1D systems mainly focused on the calculation of the self-energy and observables which can directly be computed from it. In the present paper we are interested in the grand canonical potential (GCP) from which the energy of the indirect impurity interaction can be obtained. The connection between the GCP and the 0-particle vertex (0PV) is established by replacing the normalizing grand canonical partition function $\mathscr{Z}$ in the thermodynamical average by the grand canonical partition function $\mathscr{Z}_{0}$ of the free system, that is the system without interaction and impurities. This keeps the vertex functions of order greater or equal one unaltered and merely changes the physical meaning of the $0 \mathrm{PV}$ as can be seen by looking at its definition in terms of the generating functional $\Gamma:^{24}$

$$
\Omega=\left.\Gamma(\{\phi\},\{\bar{\phi}\})\right|_{\phi=\bar{\phi}=0}=-\ln (\mathscr{Z})+\ln \left(\mathscr{Z}_{0}\right) \quad,
$$

with the external source fields $\phi$ and $\bar{\phi}$. Thus, the $0 \mathrm{PV}$ describes the difference between the GCP of the full system and the one of the free system.

For our sharp energy cutoff the flow equation of the OPV reads 19,20

$$
\partial_{\Lambda} \Omega^{\Lambda}=\frac{1}{2 \pi} \sum_{\omega= \pm \Lambda} \operatorname{Tr} \ln \left[\mathbf{1}-\Sigma^{\Lambda}(i \omega) G_{0}(i \omega)\right] e^{i \omega \eta}
$$

were the trace and the matrix structure refer to the quantum numbers and we explicitly introduced the convergence factor $e^{i \omega \eta}$, which is usually suppressed. The limit $\eta \searrow 0$ has to be taken at the end of the calculations. The validity of Eq. (16) is not restricted to the approximation scheme used here, which leads to a frequency independent self-energy.

Since in a numerical solution the flow necessarily starts from a large but finite cutoff $\Lambda_{0}$, we have to take into account the flow from $\Lambda=\infty$ to $\Lambda_{0}$ in the initial values. For the self-energy and the 2-particle vertex this procedure is described in Ref. 21. In this large $\omega$ limit on can replace $\Sigma^{\Lambda}(i \omega)$ by its frequency independent part $\Sigma^{\Lambda}$ [even if a more sophisticated truncation than the one leading to Eqs. (7)-(9) is used]. Approximating $G_{0}$ by its high frequency behavior $G_{0 ; j, j^{\prime}}(i \omega) \approx \delta_{j, j^{\prime}} /(i \omega)$ and expanding the logarithm we obtain with $\Omega^{\Lambda=\infty}=0$

$$
\Omega^{\Lambda_{0}} \approx \frac{1}{\pi} \int_{\Lambda_{0}}^{\infty} \mathrm{d} \Lambda \frac{\sin (\eta \Lambda)}{\Lambda} \operatorname{Tr} \Sigma^{\Lambda} .
$$

With the large $\Lambda$ behavior of $\Sigma^{\Lambda}$ (see Ref. 21)

$$
\Sigma_{j, j^{\prime}}^{\Lambda} \approx \Sigma_{j, j^{\prime}}^{\Lambda=\infty}+\frac{1}{\pi} \sum_{l} I_{j, l ; j^{\prime}, l} \int_{\Lambda}^{\infty} \mathrm{d} \Lambda^{\prime} \frac{\sin \left(\eta \Lambda^{\prime}\right)}{\Lambda^{\prime}}
$$

where $I_{i, j ; k, l}$ is the bare antisymmetrized interaction, one obtains performing the integrals over $\Lambda$ and $\Lambda^{\prime}$ in the limit $\eta \searrow 0$

$$
\Omega^{\Lambda_{0}} \approx \frac{1}{2} \operatorname{Tr} W+\frac{1}{8} \sum_{j, j^{\prime}} I_{j, j^{\prime} ; j, j^{\prime}} .
$$

Here $W$ denotes all single-particle terms of the Hamiltonian not taken into account in the free propagator $G_{0} \stackrel{25}{\underline{25}}$ For our problem this yields the initial values

$$
\Omega^{\Lambda_{0}}=\frac{V_{1}+V_{2}}{2}-\frac{1}{4} \sum_{j=1}^{N-1} U_{j, j+1},
$$

for site impurities and

$$
\Omega^{\Lambda_{0}}=-\frac{1}{4} \sum_{j=1}^{N-1} U_{j, j+1},
$$

for hopping impurities. After this step $\eta$ can be set to zero in Eq. (16). Using the matrix identity $\operatorname{det} e^{A}=e^{\operatorname{Tr} A}$ Eq. (16) simplifies to

$$
\partial_{\Lambda} \Omega^{\Lambda}=\frac{1}{\pi} \ln \left|\frac{\operatorname{det}\left[\tilde{G}^{\Lambda}\right]^{-1}(i \Lambda)}{\operatorname{det} G_{0}^{-1}(i \Lambda)}\right| .
$$

\section{NONINTERACTING CASE}

\section{A. Analytical results}

In the noninteracting case, we compute the energy of the indirect interaction of two site impurities by evaluating the partition function. For finite temperatures $T$ this 
gives

$$
\begin{aligned}
\frac{\mathscr{Z}}{\mathscr{Z}_{0}}=\prod_{\omega_{n}}\{ & 1-G_{0 ; j, j}\left(i \omega_{n}\right)\left[V_{1}+V_{2}\right] \\
& \left.+V_{1} V_{2}\left[G_{0 ; j, j}^{2}\left(i \omega_{n}\right)-G_{0 ; j, j+r}^{2}\left(i \omega_{n}\right)\right]\right\},
\end{aligned}
$$

where the product involves all Matsubara frequencies $\omega_{n}$ and $r \in \mathbb{N}$ denotes the distance between the two impurities. At half-filling (with chemical potential $\mu=0$ ) the
Green function of the noninteracting and impurity free system is given by

$$
G_{0 ; j, j+r}(z)=\frac{1}{\sqrt{z^{2}-4}}\left(-\frac{z}{2}+\frac{1}{2} \sqrt{z^{2}-4}\right)^{r} .
$$

The interaction energy between the impurities $V_{12}(r)$ as function of their separation $r$ can be obtained as the difference of the GCP for infinitely separated impurities and the GCP for finite $r .15$ This leads to

$$
V_{12}=-\frac{1}{\pi} \int_{0}^{\infty} \mathrm{d} \omega \ln \left|1-\frac{V_{1} V_{2} G_{0 ; j, j+r}^{2}(i \omega)}{1-G_{0 ; j, j}(i \omega)\left[V_{1}+V_{2}\right]+V_{1} V_{2} G_{0 ; j, j}^{2}(i \omega)}\right|
$$

where we took the limit $T \rightarrow 0$ and replaced the sum over Matsubara frequencies by an integral.

In the limits of weak $V_{1}, V_{2} \ll 1$ and strong $V_{1}, V_{2} \gg 1$ impurities and for sufficiently large $r$ (that is for $r \gg$ $1 / k_{F}$; throughout this work we will not be interested in the behavior of $V_{12}$ at very small separations $r \lesssim 1 / k_{F}$ ) Eq. (25) with the Green function Eq. (24) can further be evaluated. For small $V_{1}, V_{2} \ll 1$ we obtain

$$
V_{12}=(-1)^{r+1} \frac{V_{1} V_{2}}{4 \pi r}
$$

and for large $V_{1}, V_{2} \gg 1$

$$
V_{12}=\left\{\begin{array}{cc}
\frac{\pi}{6 r} & \text { for } r \text { even } \\
-\frac{\pi}{12 r} & \text { for } r \text { odd. }
\end{array}\right.
$$

In both limits the impurity interaction oscillates around zero with a periodicity of two lattice sites, which corresponds to $\pi / k_{F}$ with the Fermi momentum $k_{F}=\pi / 2$ at half filling, and decays as $1 / r$ with increasing impurity separation. For strong impurities the oscillation around zero is asymmetric, while it is symmetric for weak impurities. Furthermore, increasing the impurity strength from weak to strong for fixed $r$ the impurity interaction changes from being attractive to being repulsive (even separations) and vice versa (odd separations).

\section{B. Numerical results}

For weak and strong impurities the $r$-dependence of $V_{12}$ is shown in Figs. 2 and 3, respectively. To obtain these results we inserted the Green function Eq. (24) into Eq. (25) and performed the integral numerically (not relying on any approximations). For simplicity we chose $V_{1}=V_{2}$. To cover a wide range of separations the data are shown on a logarithmic scale. We always plot two subsequent separations. In the figures the triangles indicate a separation of an even number of lattice sites, while the circles symbolize odd separations.

We can define an effective exponent of the decay of $V_{12}$ as the logarithmic derivative of the difference of the amplitudes with respect to the separation. This exponent is shown in the insets of Figs. 2] and 3, Consistent with the asymptotic analytical results Eqs. (26) and (27) the interaction decays with the inverse of the separation. A $1 / r$ decay is also found for impurities of intermediate strength as exemplified in the inset of Fig. 4 obtained for $V_{1}=1$ and $V_{2}=4$ (for a discussion of the main part of Fig. 4, see below).

\section{Interpretation}

For weak impurities the $1 / r$ decay and the symmetric oscillations with period $\pi / k_{F}$ can be understood from the spatial dependence of the Friedel oscillations of the electron density induced by a single impurity: ${ }^{26}$ For distances sufficiently larger then $\pi / k_{F}$ the change of the density oscillates symmetrically around zero with period $\pi / k_{F}$ and in $1 \mathrm{D}$ dies off as the inverse distance from the impurity. In lowest order perturbation theory in the impurity strength this leads to a potential of similar shape at the position of the second impurity and thus to the observed behavior of $V_{12}$. This argument can also be applied to hopping impurities and we again [as in Eq. (26)] obtain a symmetrically oscillating (attractive for even $r$, repulsive for odd $r$ ) $V_{12}$ which decays as $1 / r$.

Also for strong impurities a simple picture of the $r$ dependence of $V_{12}$ can be given. To this end we consider a simplified model of a half filled tight-binding chain with open boundary conditions which follows in the limit of a single infinitely strong impurity (site or hopping). A second infinitely strong impurity is placed at distance $r$ from 


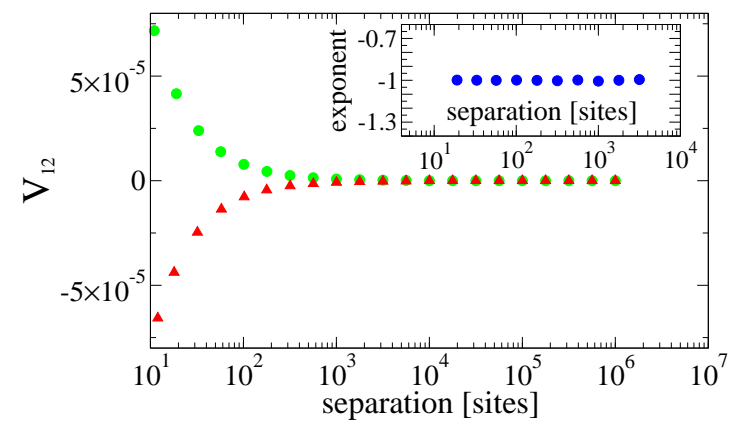

FIG. 2: (Color online) Oscillations of the impurity interaction as function of the separation for $V_{1}=V_{2}=0.1$ in the noninteracting case. Triangles: even separations; circles: odd separations. Inset: exponent of the decay.

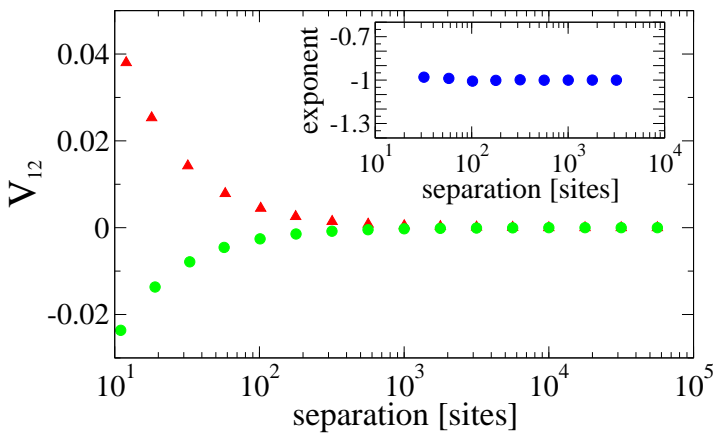

FIG. 3: (Color online) The same as in Fig. 2 but for $V_{1}=$ $V_{2}=30$.

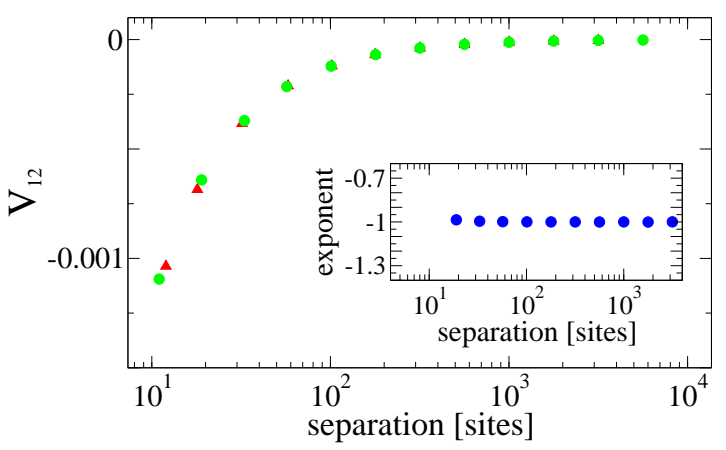

FIG. 4: (Color online) Disappearance of oscillations for $V_{1}=1$ and $V_{2}=4$ in the case of noninteracting electrons. Triangles: even separations; circles: odd separations. Inset: exponent of the decay.

the first one. The ground state energy of this setup can easily be computed analytically leading to Eq. (27) if the infinitely strong impurities are modeled as site impurities, while it gives

$$
V_{12}=\left\{\begin{array}{cl}
-\frac{\pi}{12 r} & \text { for } r \text { even } \\
\frac{\pi}{6 r} & \text { for } r \text { odd. }
\end{array}\right.
$$

for hopping impurities. We thus find that for this type of impurities the interaction $V_{12}$ is attractive (repulsive) for even (odd) separations in the limit of weak as well as of

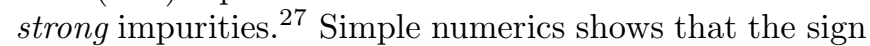
also does not change for hopping impurities of interme- diate strength.

This analysis reveals that the $1 / r$ decay as well as the prefactors $\pi / 6$ and $-\pi / 12$ follow from an even simpler consideration. $V_{12}$ can directly be extracted from the $1 / N$ correction $\Delta E$ of the groundstate energy of a tight-binding chain with open boundary conditions. A simple analytic calculation shows that $\Delta E$ is given by $\pi /(6 N)$ for odd $N$ and $-\pi /(12 N)$ for even $N$. For even $N$ the chemical potential $\mu=0$ lies between the last occupied and the first unoccupied state. In this case the results from (boundary) conformal field theory can be used. Within this approach one obtains for a model with conformal charge $c=1$

$$
\Delta E=-\pi \frac{v_{c}}{24 N},
$$

where $v_{c}$ denotes the charge velocity 28 This result also holds for $U \neq 0$. For the tight-binding model at $U=0$ one finds $v_{c}=2$ and Eq. (29) leads to $\Delta E=-\pi /(12 N)$ obtained above by direct calculation. To deduce the impurity interaction from these considerations for hopping impurities the chain length $N$ must be substituted by $r$. For site impurities a length $N$ corresponds to an impurity separation $r=N+1$. This explains the interchange of "even" and "odd" in Eqs. (27) and (28). The reason for the $1 / r$ decay of $V_{12}$ for strong impurities is thus the appearance of $1 / N$ corrections to the groundstate energy of a finite system with open boundary conditions.

Our results for $V_{12}$ in the lattice model are equivalent to the ones found in the noninteracting continuum model with $\delta$ impurities investigated in Refs. 15 and 16 . There a different interpretation is given in the limit of strong impurities.

\section{Other fillings and fine tuned parameters}

We here assumed the system to be half filled. Relaxing this does not affect the general behavior of the impurity interaction, namely the oscillation of the interaction energy decaying with the inverse separation. Merely the periodicity is altered. The behavior in the two limits of weak and strong impurities can again be understood within the two simple pictures presented above.

We next describe a behavior which we exclusively observed for site impurities and at half-filling. In a narrow crossover regime between the limits of strong and weak impurities the interaction becomes entirely attractive, but still oscillates around a decaying average value with amplitudes which scale as $1 / r$ (not shown here). A peculiar behavior is found under the constraint $V_{2}=4 / V_{1}$. For such fine tuned impurity parameters the oscillations completely disappear. The latter is depicted in the main part of Fig. 4. Note that the amplitude still decays as $1 / r$ as shown in the inset. 


\section{INTERACTING CASE}

To compute the interaction energy $V_{12}$ between the impurities for $U \neq 0$, we numerically solve the flow equations (7)-(9) and (22) with the appropriate initial values. To isolate the energy of the impurity interaction, we evaluate the GCP for different configurations, namely for $V_{1}=V_{2}=0$ yielding the extensive part of the electronic interaction, $V_{1}=0$ and $V_{2} \neq 0$ at site of interest and vice versa, finally the GCP of the full system with $V_{1} \neq 0$ and $V_{2} \neq 0$. The interaction energy $V_{12}$ is then given by

$$
\begin{aligned}
V_{12}= & \left(\Omega-\Omega_{V_{1}=V_{2}=0}\right)-\left(\Omega_{V_{1}=0 \neq V_{2}}-\Omega_{V_{1}=V_{2}=0}\right) \\
& -\left(\Omega_{V_{2}=0 \neq V_{1}}-\Omega_{V_{1}=V_{2}=0}\right) .
\end{aligned}
$$

For the case of hopping impurities the same equation holds, if one replaces $V_{\alpha}$ by $t_{\alpha}$ and keeps in mind that the impurity free case corresponds to $t_{1}=t_{2}=1$.

For $U \neq 0$ we examine systems of $2.5 \times 10^{5}$ sites, placing the impurities in the interval [22500,200000] well apart from the sections of the lattice in which the interaction is turned on and off. This enables us to calculate the interaction energy for impurity separations of up to $10^{5}$ lattice sites. We restrict ourselves to half-filling and first consider bulk interactions $U \in(0,2]$, which correspond to $\mathrm{LL}$ parameters $K \in[0.5,1)$ [see Eq. (6) ].

\section{A. Repulsive electron-electron interaction}

We focus on equal $V_{1}$ and $V_{2}$ but again verified that deviating from this restriction does not qualitatively change our main conclusions. Further down we briefly comment on the behavior for fine tuned impurity parameters as discussed at the end of the last section for $U=0$. Then it will also become important to consider $V_{1} \neq V_{2}$.

Figures 5 and 6 show the $r$ dependence of the interaction energy for weak and strong impurities. We find that the oscillation of the interaction with a periodicity of two lattice sites (corresponding to half-filling) is robust even in the presence of the electron-electron interaction. $15,16,18$ As for $U=0$ the impurity interaction for weak site impurities is repulsive for odd $r$ while it is attractive for even $r$. For strong impurities the opposite holds. Apart from the oscillation the interaction energy decreases as a function of $r$ and we extract the "effective" $r$-dependent exponent as in the last subsection. It is depicted in the insets of Figs. 5 and 6. For strong impurities a clear $1 / r$ decay is found. For weak to intermediate impurities as in Fig. 5 the exponent is different from -1 . It starts well above -1 for small $r$ and tends towards it with increasing separation.

For weak impurities $V_{1}$ and $V_{2}$ and separations $r$ sufficiently smaller then a crossover scale $r_{c}$ (see below), the indirect impurity interaction can again be understood using linear response theory. In this parameter regime the Friedel oscillations in the electron density induced by a single impurity behave as $(-1)^{r} / r^{2 K-1}, 21,29$ This leads

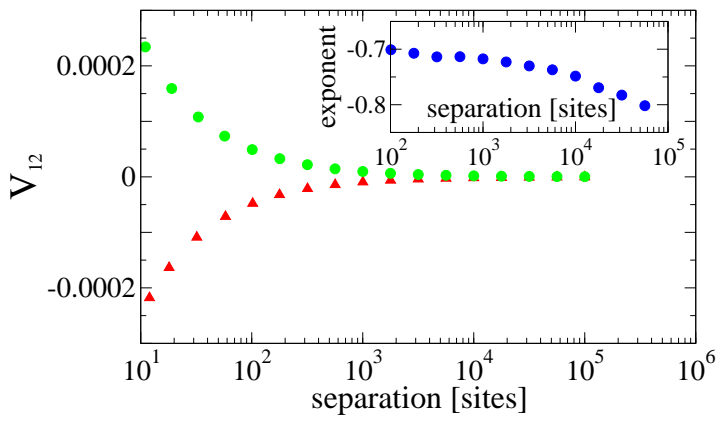

FIG. 5: (Color online) Oscillations of the interaction energy as function of the separation for $V_{1}=V_{2}=0.1$ and interaction $U=0.5$. Triangles: even separations; circles: odd separations. Inset: exponent of the decay.

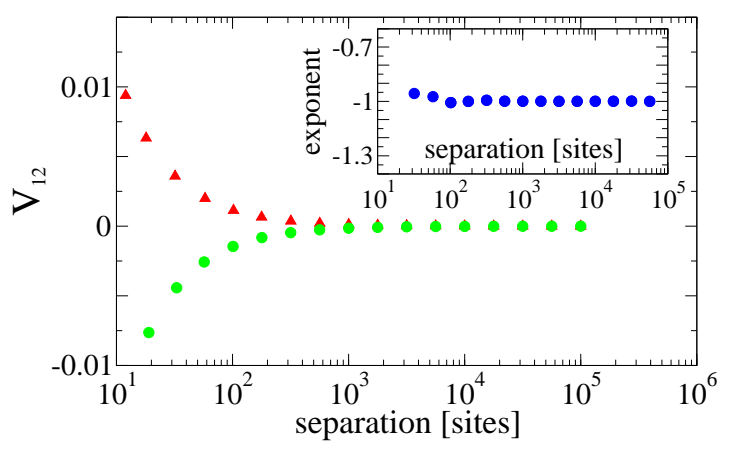

FIG. 6: (Color online) The same as in Fig. 5 but for $V_{1}=$ $V_{2}=10$.

to a potential of the same spatial dependence at the position of the second impurity. ${ }^{15}$ This argument also holds for hopping impurities. We thus expect that the oscillations of $V_{12}$ are symmetric and that the effective exponent of the decay is $1-2 K$, which corresponds to -0.72 for $U=0.5$ [see Eq. (6)]. Our results are consistent with this argument (see Figs. [5] and [7, in the latter figure $U=1$ leading to $1-2 K=-0.5)$. The small deviation of the exponent at small $r$ can be explained by our truncation of the exact hierarchy of fRG flow equations. This leads to an approximate $K_{\mathrm{fRG}}$ which is slightly smaller than the exact $K .13$

The linear response analysis cannot be used at large $V_{1}, V_{2}$ and in analogy to the $U=0$ considerations the appearance of the $1 / r$ decay as well as the oscillatory behavior can be explained in terms of the finite size scaling of the ground state energy of a system with open boundary conditions. In lowest order perturbation theory in $U, \Delta E$ is given by $-\pi(1+U / \pi) /(12 N)$ for even $N$. This is consistent with the (boundary) conformal field theory result Eq. (29) as $v_{c}(U)=2(1+U / \pi)$ to lowest order in $U$. For odd $N$ we did not succeed finding a closed form expression for the leading $U, 1 / N$ correction of the ground state energy. It is easy to show numerically that $\Delta E>0$ for odd $N$. As in the noninteracting case this explains the repulsive (attractive) impurity interactions for site impurities with even (odd) separation and attractive (repulsive) $V_{12}$ for hopping impurities with odd (even) 
$r$. We note in passing that our numerics (lowest order perturabtion theory as well as fRG) shows that for odd $N$, the $U$ dependence of $\Delta E$ cannot solely be expressed in terms of $v_{c}(U)$, as it is the case for even $N$.

For $U>0$ linear response theory in addition breaks down for $r \gg r_{c}$ even if weak bare impurities are considered. This is related to the fact that in a LL a single impurity is a relevant perturbation in the renormalization group sense. Thus, for distances sufficiently larger then $r_{c}$, that is in the low energy limit, even a single weak impurity acts as a strong perturbation (flow towards the "cut chain" fixed point) $\stackrel{3,4,5,6.7}{?}$ Consistent with this picture for $U>0$ and increasing $r$ we observe a tendency towards the exponent -1 in the decay of $V_{12}$ for weak to intermediate impurities (as in Figs. 5 and 7 ). Although the renormalization group flow of the impurity determines the scaling exponents and thus leads to the asymptotic $1 / r$ decay of $V_{12}$, it does not affect the sign of the impurity interaction. The latter is fixed by the bare impurity strength (see Figs. 5 and 6) and was discussed above. This observation shows that even for $r \gg r_{c}$ a system with weak to intermediate bare impurities is not completely equivalent to a system with strong bare impurities.

In Fig. 7 we show the effective exponent of the decay for interaction strength $U=1$ and various values of the impurity strength $V_{1}=V_{2}=V$. The complete crossover from the weak to the strong impurity behavior of the decay exponent cannot be covered for a single $V$. This follows from the $V$ and $K$ (that is $U$ ) dependence of the single-impurity crossover scale ${ }^{6}$

$$
r_{c} \propto V^{1 /(K-1)}
$$

For $V \ll 1, r_{c}$ grows exceedingly, even beyond the large system sizes we can treat using the truncated fRG. The $K$ dependence of $r_{c}$ can be read off from Fig. 8, where the effective exponent for $V_{1}=V_{2}=0.1$ and various values of the interaction strength is shown. The interaction ranges from $U=0.5$ to $U=2$, corresponding to LL parameters from $K \approx 0.86$ to $K \approx 0.5$ [see Eq. (6)]. For small to intermediate $U \leq 1$ the effective exponent starts close to the linear response result $1-2 K$ and tends towards the asymptotic value of -1 on a length scale decreasing with interaction strength. The asymptotic exponent -1 is reached for separations beyond our system size. For larger interactions $1<U \leq 2$ already at the smallest separations considered here a clear deviation from the linear response exponent is observed, while the asymptotic exponent -1 is reached for $r \sim 10^{5}$.

Similar to the noninteracting case in a narrow regime of site impurity parameters $V_{1}$ and $V_{2}$ the impurity interaction changes from being attractive (repulsive) for even (odd) $r$ to the opposite. In the crossover region $V_{12}$ becomes purely attractive and one can again fine tune $V_{1}$ and $V_{2}$ such that $V_{12}$ does not oscillate anymore.

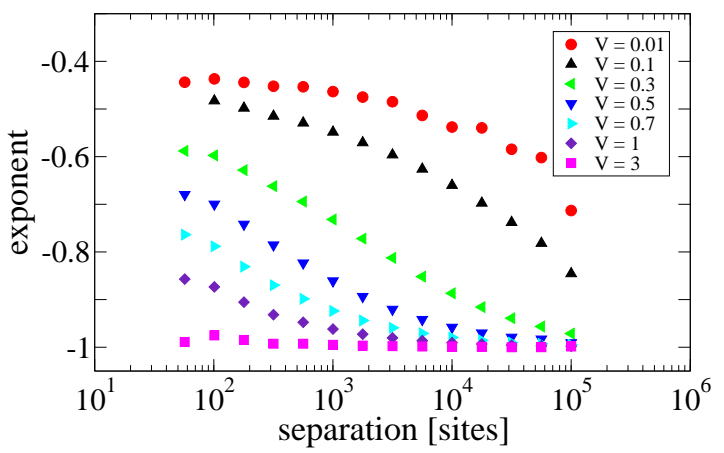

FIG. 7: (Color online) Effective exponent of the amplitude of the indirect interaction for a collection of impurity strength $V_{1}=V_{2}=V$ and interaction $U=1$. The "noise" visible in the curve for $V=0.01$ originates from the fact that $V_{12}$ is very small for small $V$.

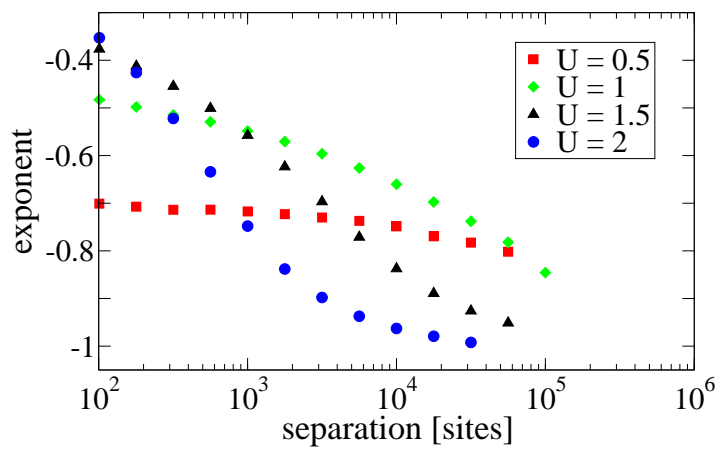

FIG. 8: (Color online) The same as in Fig. 7 but for fixed $V_{1}=V_{2}=0.1$ and different $U$.

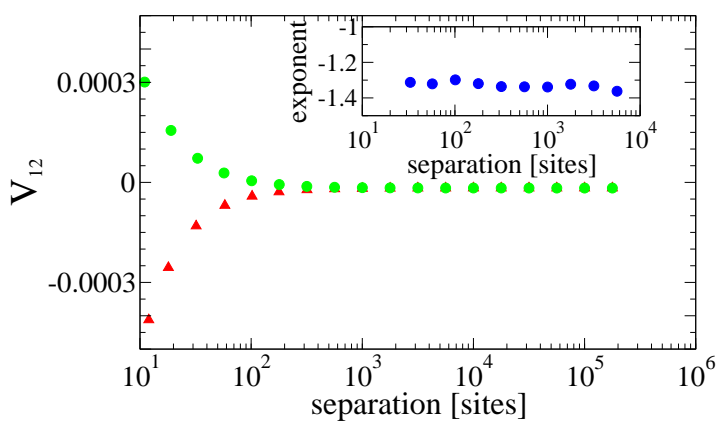

FIG. 9: (Color online) Oscillations of the interaction energy as function of the separation for site impurities $V_{1}=V_{2}=1$ and attractive interaction $U=-0.5$. Triangles: even separations; circles: odd separations. Inset: exponent of the decay. The "noise" results from the small values of $V_{12}$.

\section{B. Attractive electron-electron interaction}

Finally, we mention attractive interactions $U<0$ (that is $K>1$ ) not considered so far. In this case a single impurity is irrelevant in the renormalization group sense $e^{3,4,5,6,7}$ which suggests that regardless of the strength of the bare impurities for $r \gg r_{c}$ linear response theory can be used. We then anticipate that the impurity interaction oscillates around zero and for $r \gg r_{c}$ 
decays with the linear response exponent $1-2 K$. Our numerical results are consistent with this expectation as exemplified in Fig. 9 for site impurities with $V_{1}=V_{2}=1$ and $U=-0.5$, leading to $K(U=-1)=1.19$ and $1-2 K=-1.38$. The small deviation in the exponent again results from the approximate nature of our truncated fRG equations. A similar behavior in the decay exponent of the Friedel oscillations induced by a single impurity was discussed in Ref. 21 (see Fig. 15). For $U<0$ accurate results for the exponent of the decay of $V_{12}$ are difficult to obtain as the impurity interaction becomes exceedingly small.

\section{SUMMARY}

To summarize, we examined the indirect interaction between two impurities placed in a LL. We started from a microscopic lattice model, using the fRG in the interacting case. We observed, that for weak as well as strong impurities the interaction oscillates between being attractive and repulsive decaying algebraically with the separation of the impurities. In the case of noninteracting electrons the exponent of this decay is -1 independent of the impurity strength. For interacting electrons and weak impurity strength the powerlaw decay of $V_{12}$ starts at a larger $U$ dependent value $1-2 \mathrm{~K}$ and tends towards -1 for large separations on a scale $r_{c}$ depending on interaction and impurity strengths. For weak impurities this behavior can be understood from the oscillatory decay of Friedel oscillations of the electron density induced by an impurity using linear response theory. In the opposite limit the oscillatory $1 / r$ decay can be traced back to the finite size scaling of the ground state energy of a chain with open boundary conditions. Our approach supplements analytic calculations obtained for large interactions $\frac{18}{18}$ and those performed in the limits of large and small impurities $\frac{15,16}{1 t}$ reveals the full crossover from weak to strong impurities. For attractive interactions the impurity interaction oscillates and asymptotically (for large separations) decays with the linear response exponent $1-2 K$.

\section{Acknowledgments}

We thank J.N. Fuchs, A. Recati, and W. Zwerger for useful discussions. This work was supported by the Deutsche Forschungsgemeinschaft (SFB 602).
1 T. Giamarchi, Quantum Physics in One Dimension (Oxford University Press, New York, 2004).

${ }^{2}$ For a review see K. Schönhammer in Interacting Electrons in Low Dimensions, Ed.: D. Baeriswyl, Kluwer Academic Publishers (2005).

3 A. Luther and I. Peschel, Phys. Rev. B 9, 2911 (1974).

4 D.C. Mattis, J. Math. Phys. 15, 609 (1974).

5 W. Apel and T.M. Rice, Phys. Rev. B 26, 7063 (1982).

6 C.L. Kane and M.P.A. Fisher, Phys. Rev. Lett. 68, 1220 (1992); Phys. Rev. B 46, 15233 (1992).

7 A. Furusaki and N. Nagaosa, Phys. Rev. B 47, 4631 (1993).

8 C.L. Kane and M.P.A. Fisher, Phys. Rev. B 46, 7268 (1992).

9 A. Furusaki, Phys. Rev. B 57, 7141 (1998).

10 Y.V. Nazarov and L.I. Glazman, Phys. Rev. Lett. 91, 126804 (2003).

11 D.G. Polyakov and I.V. Gornyi, Phys. Rev. B 68, 035421 (2003).

12 V. Meden, T. Enss, S. Andergassen, W. Metzner, and K. Schönhammer, Phys. Rev. B 71, 041302(R) (2005).

13 T. Enss, V. Meden, S. Andergassen, X. Barnabé-Thériault, W. Metzner, and K. Schönhammer, Phys. Rev. B 71, 155401 (2005).

14 H. Moritz, T. Stöferle, K. Günter, M. Köhl and T. Esslinger, Phys. Rev. Lett. 94, 210401 (2005).

15 A. Recati, J.N. Fuchs, C.S. Peça, and W. Zwerger, Phys. Rev. A 72, 023616 (2005).

16 J.N. Fuchs, A. Recati, and W. Zwerger, cond-mat/0610659v2 (unpublished).

17 M.P.A. Fisher and W. Zwerger, Phys. Rev. B 32, 6190 (1985).

18 J.-S. Caux, H. Saleur, and F. Siano, Nucl. Phys. B 672,
411 (2003).

19 M. Salmhofer and C. Honerkamp, Prog. Theor. Phys. 105, 1 (2001); R. Hedden, V. Meden, Th. Pruschke, and K. Schönhammer, J. Phys.: Condensed Matter 16, 5279 (2004).

${ }^{20}$ An introduction to the fRG which only requires the knowledge of the functional integral approach to quantum many-body physics (see Ref. 24) is given in: V. Meden, lecture notes on the "Functional renormalization group", http://www.theorie.physik.uni-goettingen.de/ meden/funRG/

21 S. Andergassen, T. Enss, V. Meden, W. Metzner, U. Schollwöck, and K. Schönhammer, Phys. Rev. B 70, 075102 (2004).

22 C. N. Yang and C. P. Yang, Phys. Rev. 150, 321 (1966); ibid.150, 327 (1966); ibid.151, 258 (1966).

23 F. D. M. Haldane, Phys. Rev. B 45, 1358 (1980).

24 J. W. Negele and H. Orland, Quantum Many-Particle Systems (Addison-Wesley, Reading, 1988).

25 In the present model these are the impurity terms Eq. (4) for site impurities or Eq. (5) for hopping impurities and in addition the single-particle terms of $H_{\text {int }}$ Eq. (2).

26 See e.g. G.D. Mahan, Many-Particle Physics (Plenum Press, New York and Londo, 1990).

27 Within the reasoning of Ref. 16 this can be explained as follows: At half-filling and with hopping impurities our model is particle-hole symmetric. Thus forward scattering is forbidden by symmetry and the forward scattering phase shift $\delta$ vanishes for all $V_{1}, V_{2}$.

28 See e.g. I. Affleck and A.W.W. Ludwig, J. Phys. A: Math. Gen. 275375 (1994) and references therein.

29 R. Egger and H. Grabert, Phys. Rev. Lett. 75, 3505 (1995). 